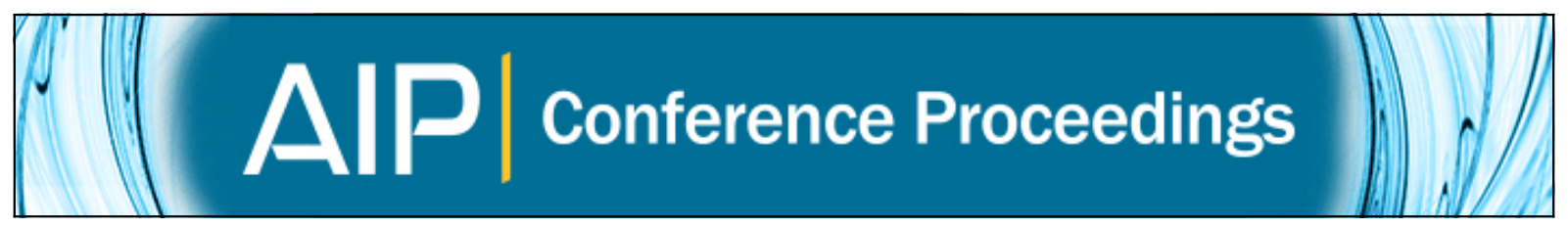

\title{
Properties of magnetic reconnection in MHD turbulence
}

S. Servidio, M. A. Shay, W. H. Matthaeus, P. Dmitruk, P. A. Cassak, and M. Wan

Citation: AIP Conference Proceedings 1216, 198 (2010); doi: 10.1063/1.3395835

View online: http://dx.doi.org/10.1063/1.3395835

View Table of Contents: http://scitation.aip.org/content/aip/proceeding/aipcp/1216?ver=pdfcov

Published by the AIP Publishing

\section{Articles you may be interested in}

Simulations of magnetic minimum variance evolution in the solar wind

AIP Conf. Proc. 1436, 302 (2012); 10.1063/1.4723623

Residual energy in MHD turbulence and in the solar wind

AIP Conf. Proc. 1436, 18 (2012); 10.1063/1.4723584

Statistical properties of solar wind discontinuities, intermittent turbulence, and rapid emergence of non-Gaussian distributions

AIP Conf. Proc. 1216, 202 (2010); 10.1063/1.3395836

Magnetic Reconnection in the Solar Wind: An Update

AIP Conf. Proc. 1216, 188 (2010); 10.1063/1.3395833

Scaling laws and intermittent structures in solar wind MHD turbulence

AIP Conf. Proc. 471, 543 (1999); 10.1063/1.58809 


\title{
Properties of magnetic reconnection in MHD turbulence
}

\author{
S. Servidio*, M.A. Shay*, W.H. Matthaeus*, P. Dmitruk ${ }^{\dagger}$, P. A. Cassak** and M. \\ $\mathrm{Wan}^{*}$ \\ *Bartol Research Institute and Department of Physics and Astronomy, University of Delaware, Newark, \\ Delaware 19716, USA \\ ${ }^{\dagger}$ Departmento de Física, Facultad de Ciencias Exactas y Naturales, Universidad de Buenos Aires, Ciudad \\ Universitaria, 1428, Buenos Aires, Argentina \\ ${ }^{* *}$ Department of Physics, West Virginia University, Morgantown, WV 26506, USA
}

\begin{abstract}
Numerical simulations of two-dimensional Magnetohydrodynamic (2D MHD) turbulence reveal the presence of a huge number of sites where magnetic reconnection locally occurs. The properties of this ensemble of reconnection events, that are spontaneously generated by turbulence, have been studied. The associated reconnection rates, computed as the electric field at the neutral points, are broadly distributed and the statistics of these events are presented. This new description of reconnection is relevant for space and laboratory plasmas, where generally turbulence is present.
\end{abstract}

Keywords: Magnetic reconnection, Magnetohydrodynamic, plasma turbulence

PACS: 52.35.Vd, 52.30.Cv, 52.35.Ra, 52.65.Kj, 52.25.Xz

\section{INTRODUCTION}

Magnetic reconnection is a nonlinear process that occurs in many astrophysical $[1,2,3,4]$ and laboratory plasmas [5]. The common feature of these nonlinear systems is the presence of an inhomogeneous magnetic field that changes rapidly across a narrow region. Reconnection, in two dimensions, implies the presence of a magnetic $X$ type neutral point where a conversion of magnetic into kinetic energy occurs $[6,7]$, and where a strong peak in the electric current density is present.

In the past decades many theoretical studies have discovered much of the basic physics of magnetic reconnection, especially in simple geometries. Sweet [6] and Parker [7], using conservation of mass, pressure balance and continuity of the electric field, revealed the essential large scale dynamics of magnetic reconnection. In particular, in the (idealized) configuration of two-dimensional Magnetohydrodynamics (2D MHD), a neutral sheet separating plasma regions is subject to a pressure imbalance that produces a plasma flow toward the neutral sheet from the strong field regions.

Generally, simulation studies of magnetic reconnection have been performed in simplified geometries and boundaries conditions $[8,9]$, but reconnection might be expected to be of importance in more general circumstances, as for example fully 2D MHD turbulence [10]. Some speculations have been made concerning both the general role of reconnection in MHD turbulence and the impact of turbulence on reconnection $[10,11,12,13,15$, 14], but no quantitative description of these effects has been documented, especially at high Reynolds numbers.
Only recently [16], it has been shown that in fully developed turbulence, complex processes of reconnection locally occur. Here we further describe the statistical features of this complex scenario of reconnection events, where initial and boundary conditions are naturally imposed by the turbulence itself.

\section{TWO-DIMENSIONAL MHD TURBULENCE}

The two-dimensional, incompressible, MHD equations in terms of a magnetic potential $a(x, y)$ and stream function $\phi(x, y)$ (uniform density $\rho=1$ ) are [17]:

$$
\begin{aligned}
& \frac{\partial \omega}{\partial t}=-(\boldsymbol{v} \cdot \nabla) \omega+(\boldsymbol{b} \cdot \nabla) j+R_{v}^{-1} \nabla^{2} \omega, \\
& \frac{\partial a}{\partial t}=-(\boldsymbol{v} \cdot \nabla) a+R_{\mu}^{-1} \nabla^{2} a
\end{aligned}
$$

with the magnetic field $\boldsymbol{b}=\nabla a \times \hat{\boldsymbol{z}}$, the velocity $\boldsymbol{v}=\nabla \phi \times$ $\hat{z}$, the current density $j=-\nabla^{2} a$, and vorticity $\omega=-\nabla^{2} \phi$. Eqs. (1)-(2) are written in familiar Alfvén units [10] with lengths scaled to a typical scale $L_{0} . R_{\mu}$ and $R_{v}$ are, respectively, magnetic and kinetic Reynolds numbers (reciprocals of kinematic viscosity and resistivity).

Eqs. (1)-(2) are solved in doubly periodic $(x, y)$ Cartesian geometry (side $\left.2 \pi L_{0}\right)$, with a dealiased (2/3-rule) pseudo-spectral code [18]. We report results from runs with up to $8192^{2}$ grid points and $R_{V}=R_{\mu}=5000$. Time integration is second order Runge-Kutta. The initial state consists of Gaussian fluctuations: random phases are employed for the initial Fourier coefficients and uncorre-

CP1216, Twelfth International Solar Wind Conference,

edited by M. Maksimovic, K. Issautier, N. Meyer-Vernet, M. Moncuquet, and F. Pantellini

(C) 2010 American Institute of Physics 978-0-7354-0759-6/10/\$30.00 
TABLE 1. Description of runs. Analysis is performed at time $t^{*}$ (peak of $\left\langle j^{2}\right\rangle$ ).

\begin{tabular}{lrrrr}
\hline & $\begin{array}{c}\text { Mesh } \\
\text { points }\end{array}$ & $\begin{array}{r}R_{\mu} \\
\left(=R_{v}\right)\end{array}$ & $\begin{array}{r}\text { Initial } \\
\text { spectrum }\end{array}$ & $t^{*}$ \\
\hline Run 1 & $4096^{2}$ & 1700 & $4 \leq k \leq 10$ & 0.4 \\
Run 2 & $4096^{2}$ & 2500 & $5 \leq k \leq 30$ & 0.2 \\
Run 3 & $4096^{2}$ & 2500 & $3 \leq k \leq 10$ & 0.7 \\
Run 4 & $8192^{2}$ & 5000 & $5 \leq k \leq 30$ & 0.3 \\
\hline
\end{tabular}

lated, equipartitioned velocity and magnetic field fluctuations are considered. For the main $8192^{2}$ run (Run 4 ), energy is initially in the shell $5 \leq k \leq 30$ ( $k$ in units of $\left.1 / L_{0}\right)$, and the total energy $E=\left\langle|\boldsymbol{v}|^{2}+|\boldsymbol{b}|^{2}\right\rangle \simeq 1(\langle\ldots\rangle$ denotes a spatial average). The time of our analysis is at the peak of the mean square current density $\left\langle j^{2}\right\rangle$, when nonlinear activity is strong $\left(t^{*} \sim 0.3\right)$. The main parameters of all the runs are reported in Table 1.

\section{ELECTRIC FIELD PROPERTIES}

We investigate the properties of the turbulent electric field, given by the Ohm's law:

$$
\boldsymbol{E}=-\boldsymbol{v} \times \boldsymbol{b}+R_{\mu}^{-1} \mathbf{j}
$$

In $2 \mathrm{D}$, only the out of plane component is present (along $z)$. In Fig. 1, color maps of the advective and diffusive terms of the electric field in Eq. (3) are separately shown. As it can be seen, the diffusive electric field $\left(\left|R_{\mu}^{-1} \mathbf{j}\right| \leq\right.$ $0.3)$ is very small compared to the $\boldsymbol{v} \times \boldsymbol{b}$-electric field $\left(\left|\mathbf{E}_{\boldsymbol{v} \times \boldsymbol{b}}\right| \leq 3\right)$. The total electric field is essentially due to the term $\boldsymbol{v} \times \boldsymbol{B}$, that is the electric field produced by fluid plasma motions. The diffusive electric field $R_{\mu}^{-1} \mathbf{j}$, is very small and with a much narrow distribution [14]. The resistive $R_{\mu}^{-1} \mathbf{j}$ contribution is non-Gaussian and gives information about the intermittent nature of MHD turbulence, and is related to the local reconnection processes.

To describe the magnetic field topology [16], we analyze $a(x, y)$ [17], and the Hessian matrix of $a, H_{i, j}^{a}(\boldsymbol{x})=$ $\frac{\partial^{2} a}{\partial x_{i} \partial x_{j}}$. At each neutral point, $\nabla a=0$, we compute the eigenvalues of $H_{i, j}^{a}$. If both eigenvalues are $>0(<0)$, this is a local minimum (maximum) of $a$ (an O-point). If the eigenvalues are of mixed sign, it is a saddle point (an $\mathrm{X}$-point). Fig. 2 shows an example of the magnetic potential with its critical points (for Run 4, for example, the number of $\mathrm{X}$-points is $\simeq 1300$.) Many magnetic islands are present, and, at the boundaries of these vortices, the diffusive electric field is bursty [see Fig. 1-(b)].

The reconnection rates are computed as the rate of change of the magnetic flux through $\partial a / \partial t$, and using
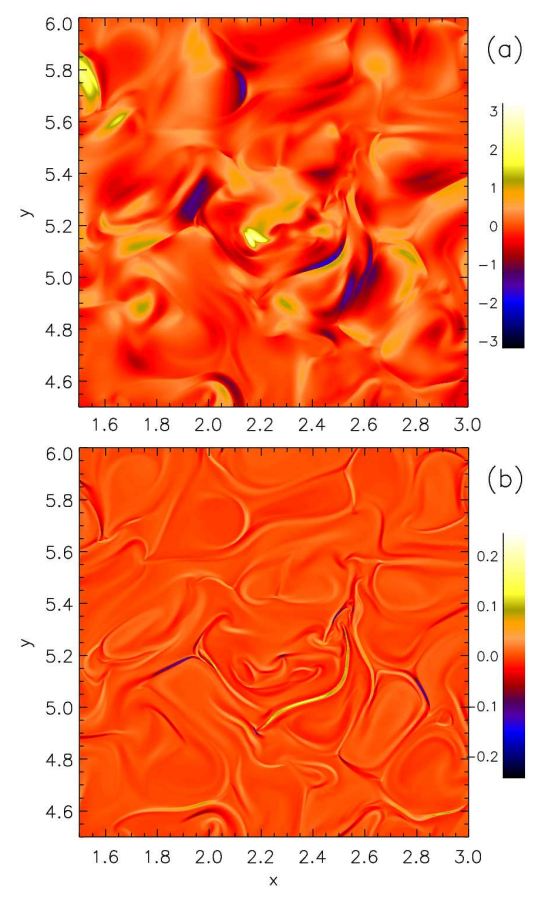

FIGURE 1. 2D plot of the $z$ components of the electric field: (a) the advective term $-\boldsymbol{v} \times \boldsymbol{b}$, and (b) the diffusive part $R_{\mu}^{-1} \boldsymbol{j}$.

Eq. (3) at the saddle points,

$$
\frac{\partial a}{\partial t}=\left.R_{\mu}^{-1} j\right|_{X-\text { point }}=-E_{\text {rec }},
$$

where $E_{r e c}$ is an abbreviation for the electric field measured at the $\mathrm{X}$-point. The reconnection rates have been normalized to the mean square fluctuation $\delta b_{r m s}^{2}$, appropriate for Alfvènic turbulence. Note that due to the orientation of the interacting pairs of magnetic islands, some of the reconnection zones have a positive reconnection rate and others, negative. In Fig. 3 the PDF of the absolute value of the reconnection rate is shown. The PDF of the electric field at the $X$-points is quite broad and peaked around zero value. The mean value of the reconnection rate is $\simeq 0.04$, with strong variations from the average. In terms of the global parameters this observed range of reconnection rates varies from very slow to fast, in fact $\left|E_{\text {rec }}\right| \in\left[10^{-6} \div 0.3\right]$. For Run $4\left(R_{\mu}=5000\right)$, the global Sweet-Parker rate would be estimated as $\sim 0.014$. In this sense the typical reconnection rate is found to be far higher than what is expected based on a simple global application of the Sweet-Parker rate $E_{r e c} \sim R_{\mu}^{-1 / 2}$. Moreover, since this rate is broadly distributed, there are some values of $E_{\text {rec }}$ that can easily exceed 0.1 , as it can be seen in Fig.s. 1 and 3). We do not attempt to further describe the functional form of this PDF as we lack a specific theoretical expectation; however, it will be of interest to attempt to explain this distribution in future work. 


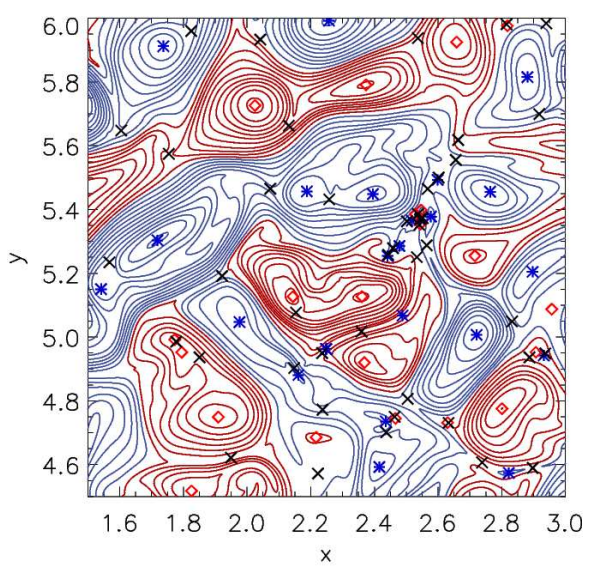

FIGURE 2. Contours of magnetic potential $a$ with the position of all the critical points: $O$-points (blue stars for the maximum and red open-circles for the minimum) and $X$-points (black $\times$ ). Only $\sim 1 / 17$ of the entire simulation box is shown.

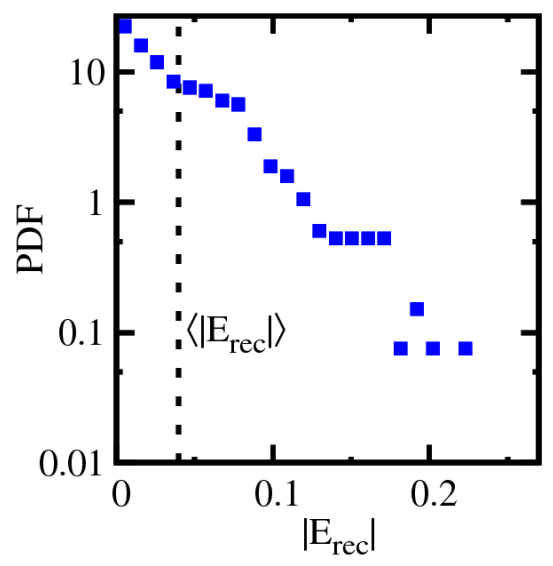

FIGURE 3. PDF of the reconnection rate (absolute value of the electric field at the X-points.) The vertical dotted black line in the panel represents the mean value of the distribution.

\section{A MODEL FOR RECONNECTION IN 2D TURBULENCE}

When magnetic reconnection is in a stationary state, the rate depends on the geometry of the diffusion region $[16,7,17]$, that can be characterized by two lengths: the thickness $\delta$ and the elongation $\ell$ of the current sheet. From the matrix $H_{i, j}^{a}(\boldsymbol{x})$ (see previous Section), the ratio $\ell / \delta \simeq \sqrt{\lambda_{\max } / \lambda_{\min }}$, being $\lambda_{\max (\min )}$ the maximum (minimum) of the eigenvalues at the X-point. Since we can obtain the ratio of the eigenvalues $\lambda_{\max } / \lambda_{\min }$, the problem reduces to measure each current sheet thickness $\delta$ (then $\ell \sim \delta \sqrt{\lambda_{\max } / \lambda_{\min } \text {.) }}$

We build up a system of reference with its origin at the $X$-point, and the (normalized) eigenvectors of the

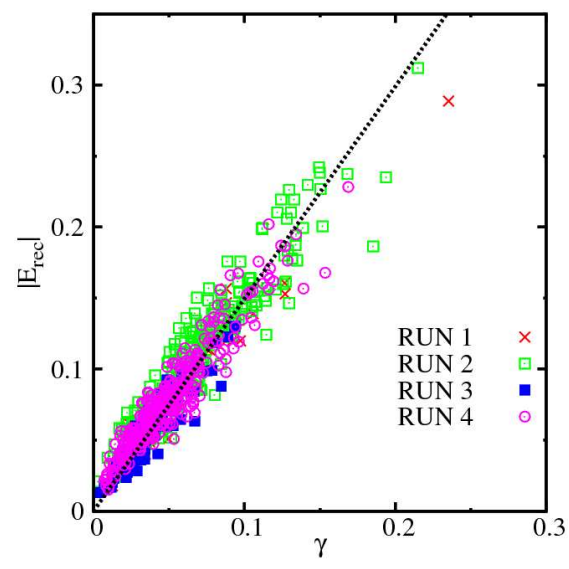

FIGURE 4. Computed reconnection rates vs. expectation from Eq. (6) [21]. Symbols distinguish runs in Table 1. The reconnection is an asymmetric Sweet-Parker scenario.

Hessian matrix identify the directions associated with inflow $\left(\hat{\boldsymbol{e}}_{s}\right)$ and outflow $\left(\hat{\boldsymbol{e}}_{l}\right)$ regions. Using this system of reference, we can obtain the tangential and the normal component of the magnetic field as $b_{t}=\hat{\boldsymbol{e}}_{l} \cdot \boldsymbol{b}$ and $b_{n}=$ $\hat{\boldsymbol{e}}_{s} \cdot \boldsymbol{b}$, respectively.

We found that the strongest reconnection events have the tangential magnetic field $b_{t}$ that reaches a maximum and then decreases going far from the saddle point $[19,20]$. This gives rise to much steeper gradients of the field near the neutral point. Another interesting feature is that reconnection in turbulence is essentially asymmetric [21]. Because of the asymmetry of the problem, we compute the total width of each current peak as $\delta=\delta_{1}+\delta_{2}$, being $\delta_{1(2)}$ the left (right) contribution in the system of reference of the X-point. The values of $\delta_{1,2}$ are found by assuming that the magnetic field, close to the $X$-point can be approximated with hyperbolic functions. We interpolate the current density $j$, along the inflow coordinate $s$, using the following parametric functions:

$$
\begin{aligned}
& f_{\text {left }}(s)=A_{1} \operatorname{sech}^{2}\left(\frac{s-s_{0}}{\delta_{1}}\right)+C_{1} \quad\left\{s<x_{0}\right\} \\
& f_{\text {right }}(s)=A_{2} \operatorname{sech}^{2}\left(\frac{s-s_{0}}{\delta_{2}}\right)+C_{2} \quad\left\{s \geq x_{0}\right\}
\end{aligned}
$$

being $A_{1,2}$ the amplitudes (they are proportional to the magnetic field inside the two islands), $s_{0}$ the position of the current peak and $C_{1,2}$ local constants. For each $\mathrm{X}$ point the fit has been optimized by an iteration procedure in order to minimized the error of the interpolation [22]. Using the above procedure, the lengths of the diffusion region ( $\delta$ and $\ell$ ) and the up-stream (tangential) magnetic fields $\left(b_{1}\right.$ and $b_{2}$ ) have been found (note we suppressed the index " $t$ ").

A recent steady, anti-parallel reconnection model [21] allows for asymmetries including unequal upstream 
magnetic field values $b_{1}$ and $b_{2}$ by analyzing conservation laws in the diffusion region. In the incompressible case the associated reconnection rate is given by:

$$
\gamma=\sqrt{\frac{b_{1}^{\frac{3}{2}} b_{2}^{\frac{3}{2}}}{R_{\mu} \ell}} .
$$

To understand scaling we evaluated Eq. (6) using several numerical experiment, listed in Table 1. Fig. 4 shows that in all the simulations the reconnection rates are consistent with the prediction given by Eq. (6). In this scenario turbulence plays a crucial role, providing locally the parameters that determine the Sweet-Parker reconnection rate: the lengths and local magnetic field strengths.

\section{CONCLUSIONS}

Properties of magnetic reconnection in turbulence have been investigated through direct, high Reynolds number, numerical simulations of 2D MHD. The turbulent cascade produces a distribution of reconnecting islands. Computing the electric field at the $X$-points, we see that turbulence produces a broad range of reconnection rates, with values in excess of 0.1 in dimensionless global Alfvén units. Only a small portion of the available turbulent electric field $\mathbf{E}_{\boldsymbol{v} \times \boldsymbol{b}}$ is supplied to the local reconnection processes. These results may explain how rapid reconnection occurs in MHD turbulence in association with the most intermittent non-Gaussian current structures. On the other hand, turbulence can also generate many reconnection sites that have very small rates.

This new perspective on reconnection may be relevant to space and astrophysical applications [2, 3, 4, 1]. On the basis of the current results, we would expect to find in a turbulent MHD system a broad distribution of size of interacting islands, with a concomitantly broad distribution of reconnection rates. A useful extension will be to employ models that are suited to low collisionality plasmas, where for example anomalous resistivity, Hall MHD, or other kinetic effects, may be important.

Note that turbulence at higher Reynolds numbers will in general have a broader range of represented dynamical length scales. Indeed, the present modest Reynolds number simulations, limited by computing resources, show thousands of reconnection zones having a range of sizes and rate. In the corona and solar wind, which presumably have much higher Reynolds numbers, one might expect even more reconnection zones and with a greater range of scales and rates.

Finally we remark that extensions of this approach to the three dimensional case (3D) would of course be desirable, and could produce valuable insights into real systems. However, due to the enormous computational requirements, lack of applicable methodologies (e.g., an extension of the Hessian approach) and the inherent complexities of 3D reconnection [23], such efforts make take some time to be realized.

This research is supported by NSF ATM-0539995, (SHINE) ATM-0752135, NASA NNG06GD47G, NNX07AR48G and (Heliophysics Theory) NNX08AI47G. P. D. is a member of Carrera Investigador Cientifico of CONICET.

\section{REFERENCES}

1. E. N. Parker, Astrophys. J. 264, pp. 642-647 (1983).

2. A. Retinò, D. Sundkvist, A. Vaivads, F. Mozer, M. André, and C. J. Owen, Nature Physics 3, pp. 236-238 (2007).

3. J. T. Gosling, and A. Szabo, J. Geophys. Res. 113, A10103 (2008).

4. D. Sundkvist, A. Retinò, A. Vaivads, and S. D. Bale, Phys. Rev. Lett. 99, pp. 025004-025004-4 (2007).

5. J. B. Taylor, Rev. Mod. Phys. 58, pp. 741-763 (1986).

6. P. A. Sweet, Electromagnetic Phenomena in Cosmical Physics, Cambridge Univ. Press, N.Y. 1958, p. 123.

7. E. N. Parker, J. Geophys. Res. 62, 509-520 (1957).

8. T. Sato, T. Hayashi, T. Tamao, and A. Hasegawa Phys. Rev. Lett. 41, pp. 1548-1551 (1978).

9. J. Birn, J. F. Drake, M. A. Shay, B. N. Rogers, R. E. Denton, M. Hesse, M. Kuznetsova, Z. W. Ma, A. Bhattacharjee, A. Otto and P. L. Pritchett, J. Geophys. Res. 106, pp. 3715-3720 (2001).

10. W. H. Matthaeus and D. Montgomery, Ann. N.Y. Acad. Sci. 357, pp. 203-222 (1980).

11. W. H. Matthaeus and S. L. Lamkin, Phys. Fluids 29, pp. 2513-2534 (1986).

12. H. R. Strauss, Astrophys. J. 326, pp. 412-417 (1988).

13. V. Carbone, P. Veltri and A. Mageney, Phys. Fluids A 2, pp. 1487-1496 (1990).

14. P. Dmitruk, and W. H. Matthaeus, Phys. Plasmas 13, pp. 042307-042307-8 (2006).

15. G. Kowal1, A. Lazarian, E. T. Vishniac, and K. Otmianowska-Mazur, Astrophys. J. 700, pp. 63-85 (2009).

16. S. Servidio, W. H. Matthaeus, M. A. Shay, P. A. Cassak and P. Dmitruk, Phys. Rev. Lett. 102, 115003-115003-4 (2009).

17. D. Biskamp, Magnetic Reconnection in Plasmas, Cambridge Univ. Press, Cambridge, 2000, pp. 5-202.

18. S. Ghosh, M. Hossain, and W. H. Matthaeus, Comput. Phys. Commun. 74, pp. 18-40 (1993).

19. J. D. Craig and P. G. Watson, Astrophys. J. 516, pp. 924-933 (1999).

20. J. C. Dorelli and J. Birn, J. Geophys. Res. 108, pp. 1183 (2003).

21. P. A. Cassak and M. A. Shay, Phys. Plasmas 14, pp. 102114-102114-11 (2007).

22. W. H. Press, B. P. Flannery, and S. A. Teukolsky, Numerical Recipes, Cambridge Univ. Press, New York, 1986, pp. 387-436.

23. R. Schindler, M. Hesse and J. Birn, J. Geophys. Res., 93, 5547, (1988); J. Birn, M. Hesse and K. Schindler, Adv. Space. Res., 19, 1763 (1997). 